\title{
Silica supported yttrium trinitrate: preparation, characterization and application in catalytic Biginelli condensation
}

\author{
Mohabbat Havaei $^{\mathrm{a}^{*}}$, Bahador Karami ${ }^{\mathrm{a}}$ and Saeed Khodabakhshi ${ }^{\mathrm{b}}$
}

${ }^{a}$ Department of Chemistry, Yasouj University, Yasouj, Zip Code: 75918-74831 P.O.Box 353, Iran ${ }^{b}$ Nanotechnology Research Center, Research Institute of Petroleum Industry, Tehran, Iran

CHRONICLE ABSTRACT

Article history:

Received January 22, 2014

Received in revised form

February 02, 2014

Accepted 8 May 2014

Available online

9 May 2014

\section{Keywords:}

Silica supported yttrium trinitrate

Solvent-free

Biginelli condensation
New silica-supported yttrium trinitrate was prepared, characterized, and used as a reusable catalyst for the three-component synthesis of quinazolinones/thiones via the Biginelli condensation under solvent-free conditions. Non-toxic reagent, short reaction times, simple work-up procedure, and high yield of the products are some of the important advantages of present method.

\section{Introduction}

Design and preparation of solid phase supported catalysts have attracted a considerable attention. ${ }^{1-3}$ Among solid supports used for catalysts preparation, catalysts which are include charcoal, ${ }^{4}$ alumina, ${ }^{5-7}$ and polymer, ${ }^{8,9}$ silica affords some advantages such as good mechanical and thermal stabilities as well as ease of scalability. Many silica supported catalysts brings an aditional benefit decreasing a reactor corrosion and increase an environmentally safety. ${ }^{10,11}$

The chemistry of some heterocyclic compounds such as quinazoline system has received an increasing interest because of its biological significance. ${ }^{12}$

The most general method for the synthesis of octahydroquinazolinones/thiones involves the onepot Biginelli reaction of cyclic 1,3-diones with aromatic aldehydes and urea/thiourea in the presence of different Lewis or Brønsted acids. Nevertheless, most of the present procedures have disadvantages ${ }^{13-18}$ such as the use of strongly acidic conditions or organic solvents, ${ }^{13,14}$ unsatisfactory yields, ${ }^{15}$ the use of non-recyclable catalysts, ${ }^{16}$ and long reaction times. ${ }^{14,15}$

\footnotetext{
* Corresponding author. Fax: (+98) 7412242167; Tel: (+98)7412223048

E-mail address: karami@mail.yu.ac.ir (B. Karami) 


\section{Results and discussion}

As part of our program aimed at developing novel and green solid acid catalysts, recently, we have reported some procedures for the preparation of silica tungstic acid (STA), ${ }^{19}$ silica supported zirconium oxychloride $\left(\mathrm{ZrOCl}_{2} \cdot 8 \mathrm{H}_{2} \mathrm{O} / \mathrm{SiO}_{2}\right),{ }^{20}$ molybdate sulfuric acid (MSA), ${ }^{21}$ and tungstate sulfuric acid (TSA). ${ }^{22,23}$

Based on recently published works, suggesting the use of yttrium nitrate hexahydrate as an efficient catalyst, ${ }^{24,25,26}$ we used it as a catalyst, but the reactions proceeded sluggishly. Beyond this, we found that a low amount of the catalyst is recoverable. In order to optimize the reaction conditions, the $\mathrm{Y}\left(\mathrm{NO}_{3}\right)_{3} \cdot \mathrm{nH}_{2} \mathrm{O} / \mathrm{SiO}_{2}$ was prepared and characterized by $\mathrm{X}$-ray fluorescence, $\mathrm{X}$-ray diffraction, and Fourier transform infrared spectroscopy. Fig. 1 shows the $\mathrm{X}$-ray diffraction patterns (XRD) for $\mathrm{Y}\left(\mathrm{NO}_{3}\right)_{3} \cdot \mathrm{nH}_{2} \mathrm{O} / \mathrm{SiO}_{2}$.

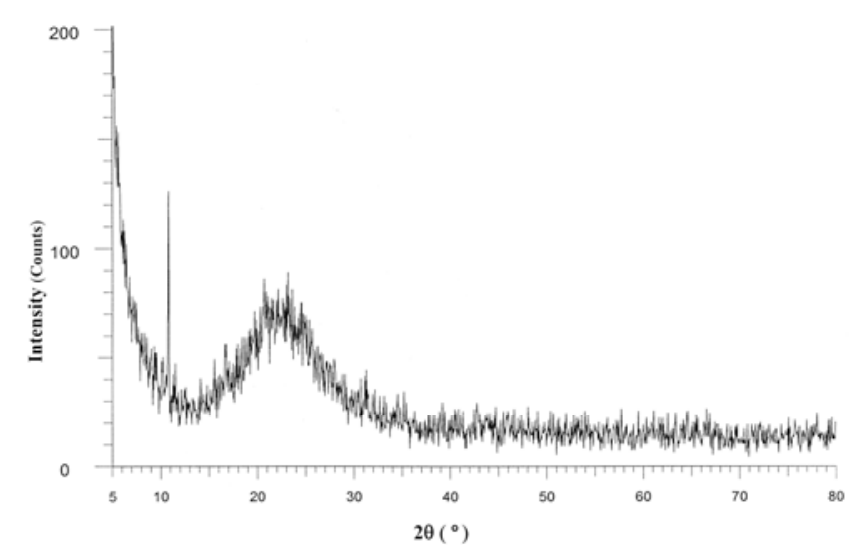

Fig. 1. $\mathrm{XRD}$ pattern for $\mathrm{Y}\left(\mathrm{NO}_{3}\right)_{3} \cdot \mathrm{nH}_{2} \mathrm{O} / \mathrm{SiO}_{2}$

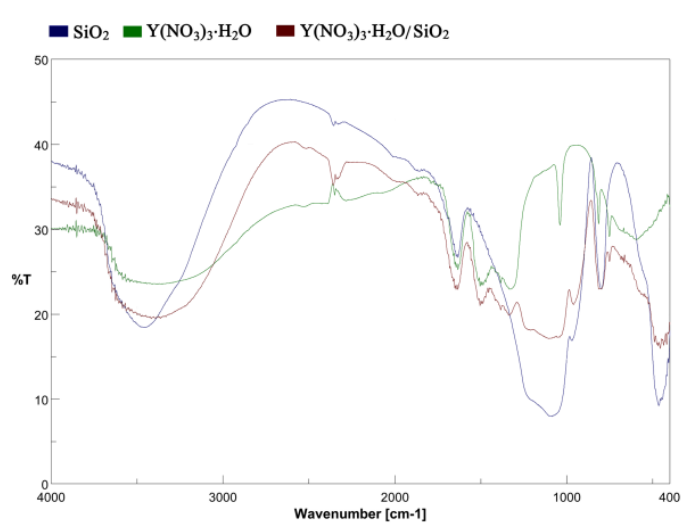

Fig. 2. FT-IR spectrum of $\mathrm{SiO}_{2}, \mathrm{Y}\left(\mathrm{NO}_{3}\right)_{3} \cdot 6 \mathrm{H}_{2} \mathrm{O}$, and $\mathrm{Y}\left(\mathrm{NO}_{3}\right)_{3} \mathrm{nH}_{2} \mathrm{O} / \mathrm{SiO}_{2}$

According to the published reports, ${ }^{27,28}$ the XRD spectrum of $\mathrm{Y}\left(\mathrm{NO}_{3}\right)_{3} \mathrm{nH}_{2} \mathrm{O} / \mathrm{SiO}_{2}$ showed that this material is amorphous due to the amorphous nature of silica gel. Therefore, no distinct peaks are observed in the sample except for the harrow-like pattern at $2 \theta$ degree ( $\theta$ is the Bragg's angle) between $15^{\circ}-30^{\circ}$ (attributed to amorphous silica gel) and a sharp peak at $11^{\circ}(2 \theta)$ from the smaller inset (attributed to insertion of $\mathrm{Y}$ (III) into the framework of amorphous $\mathrm{SiO}_{2}$ ). In addition, the XRF data of $\mathrm{Y}\left(\mathrm{NO}_{3}\right)_{3} \mathrm{nH}_{2} \mathrm{O} / \mathrm{SiO}_{2}$ indicate the presence of yttrium as $0.696 \% \mathrm{~W} / \mathrm{W}$ (Table 1).

Table 1. XRF data for $\mathrm{Y}\left(\mathrm{NO}_{3}\right)_{3} \mathrm{nH}_{2} \mathrm{O} / \mathrm{SiO}_{2}$.

\begin{tabular}{ccc}
\hline Entry & Compounds & Concentration $(\% \mathrm{~W} / \mathrm{W})$ \\
\hline 1 & $\mathrm{SiO}_{2}$ & 99.168 \\
3 & $\mathrm{Y}$ & 0.696 \\
4 & $\mathrm{MgO}$ & 0.070 \\
5 & $\mathrm{SO}_{3}$ & 0.066 \\
\hline
\end{tabular}

The FT-IR spectrum of $\mathrm{Y}\left(\mathrm{NO}_{3}\right)_{3} 6 \mathrm{H}_{2} \mathrm{O}, \mathrm{SiO}_{2}$, and $\mathrm{Y}\left(\mathrm{NO}_{3}\right)_{3} \mathrm{nH}_{2} \mathrm{O} / \mathrm{SiO}_{2}$ are shown in Fig. 2. The spectrum of $\mathrm{Y}\left(\mathrm{NO}_{3}\right)_{3} \cdot \mathrm{nH}_{2} \mathrm{O} / \mathrm{SiO}_{2}$ shows the characteristic bonds for $\mathrm{Y}\left(\mathrm{NO}_{3}\right)_{3} \cdot 6 \mathrm{H}_{2} \mathrm{O}$ and $\mathrm{SiO}_{2}$.

The absorptions at 3600-3300 (broad), 1650 (sharp), 1500 (sharp), and 1300 (sharp) $\mathrm{cm}^{-1}$ in the catalyst spectrum reveal both bonds in anhydrous $\mathrm{Y}\left(\mathrm{NO}_{3}\right)_{3}$ and $\mathrm{SiO}_{2}$. After preparation and characterization, we employed the $\mathrm{Y}\left(\mathrm{NO}_{3}\right)_{3} \mathrm{nH}_{2} \mathrm{O} / \mathrm{SiO}_{2}$ for the solvent-free synthesis of quinazolinones/thiones $\mathbf{4}$ via the Biginelli reaction of aromatic aldehydes $\mathbf{1}$ with cyclic 1,3-diones 2 and urea/thiourea $\mathbf{3}$. The reactions were efficiently catalyzed to yield the desired product $\mathbf{4}$ in good to excellent yields without the formation of octahydoxanthenes $5 .^{29}$ 
<smiles>[R]C1([R])CC(=O)C2=C(C1)OC1=C(C(=O)CC([R])([R])C1)C2[Al]</smiles>

5<smiles>[X]C(N)=[V]C(=O)[Al]</smiles>

3 (i)<smiles>[X]C1=NC([Al])C2=C(CC([R])([R])CC2=O)N1</smiles>

4

(i): $\mathrm{Y}\left(\mathrm{NO}_{3}\right)_{3} \cdot 6 \mathrm{H}_{2} \mathrm{O}$, solvent-free, $100{ }^{\circ} \mathrm{C}$

$\mathrm{R}$ : $\mathrm{H}$ or $\mathrm{Me}$

$\mathrm{X}: \mathrm{O}$ or $\mathrm{S}$

Scheme 1. Synthesis of quinazolinones/thiones using $\mathrm{Y}\left(\mathrm{NO}_{3}\right)_{3} \cdot \mathrm{nH}_{2} \mathrm{O} / \mathrm{SiO}_{2}$.

A brief investigation on the reaction conditions was carried out through the synthesis of model compound 4a. A catalyst-free reaction was performed and the reaction was not completed even at a high temperature after a long reaction time. After applying various amounts of catalyst, and a wide range of temperatures (Table 2), it was found that the condensation reaction can be efficiently carried out by adding $0.004 \% \mathrm{~W} / \mathrm{W}$ of the catalyst at $80{ }^{\circ} \mathrm{C}$ under solvent-free conditions in a short time span of $20 \mathrm{~min}$. Increasing the catalyst amount did not affect the progress of the reaction markedly.

Table 2. Optimization of $\mathrm{Y}\left(\mathrm{NO}_{3}\right)_{3} \mathrm{nH}_{2} \mathrm{O} / \mathrm{SiO}_{2}$ in the synthesis of $\mathbf{4 a}$ at $100{ }^{\circ} \mathrm{C}$ under solvent-free conditions

\begin{tabular}{cccc}
\hline Entry & Catalyst $(\% \mathrm{~W} / \mathrm{W}) /$ Temperature $\left({ }^{\circ} \mathrm{C}\right)$ & Time $(\min )$ & Yield $(\%)$ \\
\hline 1 & $-/ 25$ & 480 & - \\
2 & $-/ 100$ & 480 & 25 \\
3 & $0.0013 / 25$ & 480 & trace \\
4 & $0.0013 / 100$ & 480 & 95 \\
5 & $0.0040 / 25$ & 480 & 50 \\
6 & $0.0040 / 100$ & 20 & 94 \\
7 & $0.0066 / 100$ & 80 & 84 \\
8 & $0.0132 / 100$ & 120 & 85 \\
\hline
\end{tabular}

After evaluation and screening, the scope of the reaction was examined under the optimized conditions (Table 3). It was observed that the process can tolerate both electron-withdrawing and electron-donating group present in benzaldehydes and quinazolinones/thions were formed in high to exellent yields.

The recyclable catalysts improve both the environmental impact and the economic profile of the current method. The $\mathrm{Y}\left(\mathrm{NO}_{3}\right)_{3} \mathrm{nH}_{2} \mathrm{O} / \mathrm{SiO}_{2}$ was easily separated from the reaction mixture by filtering and drying and reused three times without significant loss of activity (Fig. 3).

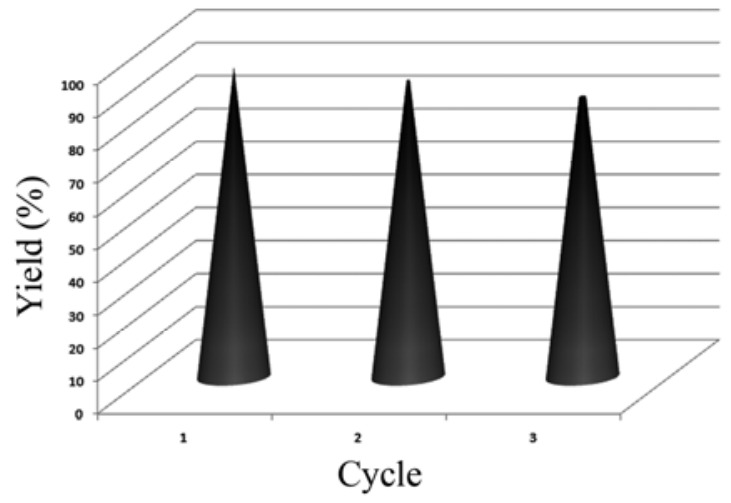

Fig. 3. Recyclability of the $\mathrm{Y}\left(\mathrm{NO}_{3}\right)_{3} \mathrm{nH}_{2} \mathrm{O} / \mathrm{SiO}_{2}$ as catalyst in the synthesis of $4 \mathbf{a}$ at $100{ }^{\circ} \mathrm{C}$ under solvent-free conditions (reaction time $=20-25 \mathrm{~min}$ ) 
Table 3. Synthesis of quinazolinones/thiones 4 via the Biginelli method in the presence of $\mathrm{Y}\left(\mathrm{NO}_{3}\right)_{3} \cdot \mathrm{nH}_{2} \mathrm{O} / \mathrm{SiO}_{2}$ at $100{ }^{\circ} \mathrm{C}$ under solvent-free conditions

\begin{tabular}{|c|c|c|c|c|c|c|}
\hline Entry & $\mathrm{R}$ & Aromatic & $\mathrm{X}$ & Time $(\min )$ & Yield $^{a}(\%)$ & M.p. $\left({ }^{\circ} \mathrm{C}\right)$ \\
\hline $4 a$ & $\mathrm{Me}$ & $\mathrm{C}_{6} \mathrm{H}_{5}$ & $\mathrm{O}$ & 20 & 94 & $288-290$ \\
\hline $4 \mathbf{b}$ & $\mathrm{Me}$ & $4-\mathrm{MeO}-\mathrm{C}_{6} \mathrm{H}_{4}$ & $\mathrm{O}$ & 10 & 88 & $272-274$ \\
\hline 4c & $\mathrm{Me}$ & $2-\mathrm{Cl}-\mathrm{C}_{6} \mathrm{H}_{4}$ & $\mathrm{O}$ & 25 & 85 & $271-273$ \\
\hline 4d & $\mathrm{Me}$ & $4-\mathrm{Br}-\mathrm{C}_{6} \mathrm{H}_{4}$ & $\mathrm{O}$ & 20 & 85 & $324-326$ \\
\hline $4 f$ & $\mathrm{Me}$ & $4-\mathrm{F}-\mathrm{C}_{6} \mathrm{H}_{4}$ & $\mathrm{O}$ & 25 & 80 & $300-302$ \\
\hline $4 g$ & $\mathrm{Me}$ & $2-\mathrm{MeO}-\mathrm{C}_{6} \mathrm{H}_{4}$ & $\mathrm{O}$ & 25 & 85 & 197-199 \\
\hline $4 h$ & $\mathrm{Me}$ & $4-\mathrm{Me}-\mathrm{C}_{6} \mathrm{H}_{4}$ & $\mathrm{O}$ & 30 & 90 & $300-302$ \\
\hline $4 \mathbf{i}$ & $\mathrm{Me}$ & $3-\mathrm{O}_{2} \mathrm{~N}-\mathrm{C}_{6} \mathrm{H}_{4}$ & $\mathrm{O}$ & 15 & 90 & 297-299 \\
\hline $\mathbf{4 j}$ & $\mathrm{H}$ & $\mathrm{C}_{6} \mathrm{H}_{5}$ & $\mathrm{O}$ & 45 & 95 & $275-277$ \\
\hline $4 \mathbf{k}$ & $\mathrm{H}$ & $4-\mathrm{Cl}-3-\mathrm{O}_{2} \mathrm{~N}-\mathrm{C}_{6} \mathrm{H}_{3}$ & $\mathrm{O}$ & 15 & 90 & $209-211$ \\
\hline 41 & $\mathrm{H}$ & 4-Br- $\mathrm{C}_{6} \mathrm{H}_{4}$ & $\mathrm{O}$ & 20 & 85 & $275-277$ \\
\hline $4 m$ & $\mathrm{H}$ & $4-\mathrm{Cl}-\mathrm{C}_{6} \mathrm{H}_{4}$ & $\mathrm{O}$ & 25 & 90 & $281-282$ \\
\hline $4 n$ & $\mathrm{H}$ & $2-\mathrm{MeO}-\mathrm{C}_{6} \mathrm{H}_{4}$ & $\mathrm{O}$ & 35 & 85 & 197-199 \\
\hline 40 & $\mathrm{Me}$ & $2,4-\mathrm{Cl}_{2}-\mathrm{C}_{6} \mathrm{H}_{3}$ & $\mathrm{O}$ & 20 & 85 & $263-265$ \\
\hline $4 p$ & $\mathrm{Me}$ & $3-\mathrm{Br}-\mathrm{C}_{6} \mathrm{H}_{4}$ & $\mathrm{O}$ & 25 & 93 & $265-267$ \\
\hline $\mathbf{4 q}$ & $\mathrm{Me}$ & $3-\mathrm{Cl}-\mathrm{C}_{6} \mathrm{H}_{4}$ & $\mathrm{O}$ & 20 & 85 & $290-292$ \\
\hline $4 r$ & $\mathrm{Me}$ & 4-Me- $\mathrm{C}_{6} \mathrm{H}_{4}$ & $\mathrm{~S}$ & 25 & 88 & $280-282$ \\
\hline $4 s$ & $\mathrm{Me}$ & $4-\mathrm{Br}-\mathrm{C}_{6} \mathrm{H}_{4}$ & $\mathrm{~S}$ & 30 & 80 & $290-292$ \\
\hline $4 t$ & $\mathrm{Me}$ & $4-\mathrm{MeO}-\mathrm{C}_{6} \mathrm{H}_{4}$ & $\mathrm{~S}$ & 20 & 85 & $268-270$ \\
\hline $4 u$ & $\mathrm{Me}$ & $\mathrm{C}_{6} \mathrm{H}_{5}$ & $\mathrm{~S}$ & 25 & 87 & $280-282$ \\
\hline
\end{tabular}

Table 4. Synthesis of 4a under various reaction conditions.

\begin{tabular}{|c|c|c|c|}
\hline Entry & Conditions & Time $(\min )$ & Yield $^{\mathrm{a}}(\%)^{\text {Ref. }}$ \\
\hline 1 & Conc. $\mathrm{H}_{2} \mathrm{SO}_{4} / \mathrm{EtOH} / 80$ & 540 & -12 \\
\hline 2 & Conc. $\mathrm{H}_{2} \mathrm{SO}_{4} / \mathrm{H}_{2} \mathrm{O} / 25$ & 180 & $85^{13}$ \\
\hline 3 & $\mathrm{HClO}_{4}-\mathrm{SiO}(0.05 \mathrm{~g}) / \mathrm{CH}_{3} \mathrm{CN} / \mathrm{Reflux}$ & 360 & $54^{14}$ \\
\hline 4 & Conc. $\mathrm{HCl} / \mathrm{EtOH} / \mathrm{Reflux}$ & 390 & -15 \\
\hline 5 & Acid Alumina/Neat/Reflux & 360 & -17 \\
\hline 6 & $\mathrm{TMSCl} / \mathrm{CH}_{3} \mathrm{CN} / \mathrm{Reflux}$ & 300 & $15^{13}$ \\
\hline 7 & $\mathrm{TMSCl} /\left(\mathrm{CH}_{3} \mathrm{CN} / \mathrm{DMF}\right) / \mathrm{Reflux}$ & 90 & $95^{13}$ \\
\hline 8 & $\mathrm{VCl}_{3} / \mathrm{CH}_{3} \mathrm{CN} / \mathrm{Reflux}$ & 120 & $92^{16}$ \\
\hline 9 & $\mathrm{SiO}_{2}(0.03 \mathrm{~g}) /$ Solvent-Free $/ 100$ & 360 & $25^{\text {This work }}$ \\
\hline 10 & $\mathrm{Y}\left(\mathrm{NO}_{3}\right)_{3} \cdot \mathrm{nH}_{2} \mathrm{O}(0.003 \mathrm{~g}) /$ Solvent-Free $/ 100$ & 300 & $90^{\text {This work }}$ \\
\hline 11 & $\mathrm{Y}\left(\mathrm{NO}_{3}\right)_{3} \cdot \mathrm{nH}_{2} \mathrm{O} / \mathrm{SiO}_{2}(0.03 \mathrm{~g}) /$ Solvent-Free $/ 100$ & 20 & $94^{\text {This work }}$ \\
\hline
\end{tabular}

A plausible mechanism for the $\mathrm{Y}\left(\mathrm{NO}_{3}\right)_{3} \cdot \mathrm{nH}_{2} \mathrm{O} / \mathrm{SiO}_{2}$-catalyzed synthesis of $\mathbf{4}$ has been proposed as shown in Scheme 2. Y (III) acts as a Lewis acid and activates the carbonyls, thus, will decreases the energy of the transition state and increases the rate of the nucleophilic displacement. $\mathrm{Y}\left(\mathrm{NO}_{3}\right)_{3} \mathrm{nH}_{2} \mathrm{O} / \mathrm{SiO}_{2}$ also plays an important role in dehydration steps.

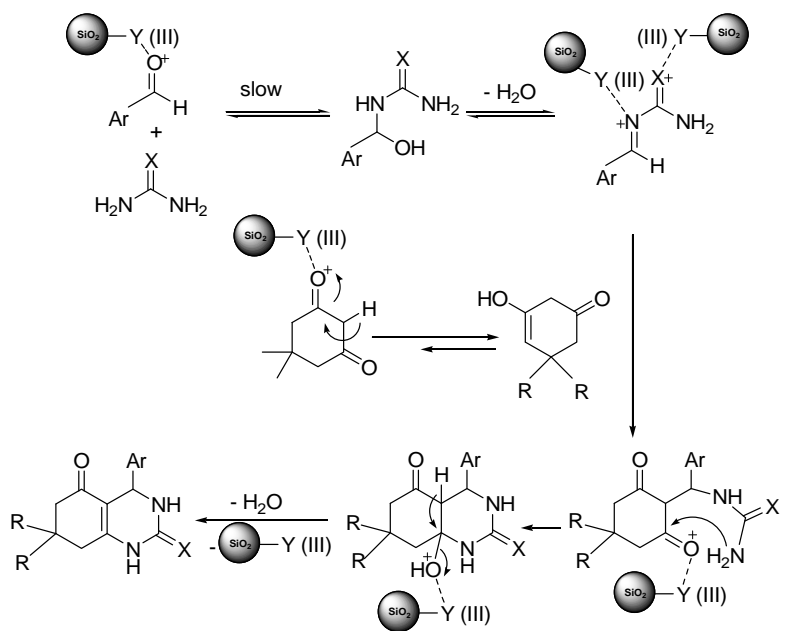

Scheme 2. Proposed mechanism for the synthesis of 4 using $\mathrm{Y}\left(\mathrm{NO}_{3}\right)_{3} \mathrm{nH}_{2} \mathrm{O} / \mathrm{SiO}_{2}$ 


\section{Conclusions}

In summary, the silica-supported yttrium (III) was judiciously used as a catalyst for the solventfree synthesis of quinazolinones/thions via the Biginelli reaction. The $\mathrm{Y}\left(\mathrm{NO}_{3}\right)_{3} \cdot \mathrm{nH}_{2} \mathrm{O} / \mathrm{SiO}_{2}$ is recyclable, safe, and thermally stable. This method may have some advantages over the previously reported ones, there are the use of a safe and recyclable catalyst, avoidance of toxic solvents, high product yields, short reaction times, and an easy work-up procedure.

\section{Experimental}

\subsection{Materials and Methods}

The chemicals were purchased from Merck and Aldrich chemical companies. The reactions were monitored by TLC (silica-gel $60 \mathrm{~F}_{254}$, hexane: EtOAc). IR spectra (in $\mathrm{KBr}$ discs) were recorded on a FTIR Shimadzu-470 spectrometer. Melting points were determined on a KRUSS model instrument. ${ }^{1} \mathrm{H}$ NMR spectra were recorded on a Bruker Avance II $400 \mathrm{NMR}$ spectrometer at $400 \mathrm{MHz}$, in which $\mathrm{CDCl}_{3}$ was used as solvent and TMS as the internal standard. X-ray diffraction (XRD) pattern was obtained by Philips $\mathrm{X}$ Pert Pro $\mathrm{X}$ diffractometer operated with a Ni-filtered $\mathrm{Cu} \mathrm{K \alpha}$ radiation source. X-ray fluorescence (XRF) spectroscopy was recorded by X-Ray Fluorescence Analyzer, Bruker, $\mathrm{S}_{4}$ Pioneer, Germany.

\subsection{Preparation of catalyst}

The grafted silica gel $(4 \mathrm{~g})$ was stirred with yttrium nitrate hexahydrate $(0.766 \mathrm{~g}, 0.2 \mathrm{mmol})$ in $\mathrm{CHCl}_{3}(10 \mathrm{~mL})$ under reflux condition for $2 \mathrm{~h}$. The mixture was then filtered, washed thoroughly with chloroform $(3 \times 10 \mathrm{ml})$, and dried.

\subsection{Preparation of quinazolinones/thiones 4}

A mixture of urea/thiourea $(1.2 \mathrm{mmol})$, aromatic aldehyde $(1 \mathrm{mmol})$, cyclic 1,3 -dione $(1 \mathrm{mmol})$, and $\mathrm{Y}\left(\mathrm{NO}_{3}\right)_{3} \cdot \mathrm{nH}_{2} \mathrm{O} / \mathrm{SiO}_{2}(0.03 \mathrm{~g})$ was stirred with small Teflon sickle blades and heated at $100{ }^{\circ} \mathrm{C}$ in a preheated oil bath for an appropriate time. After completion of the reaction as indicated by TLC, the reaction mixture was cooled to room temperature and dichloromethane $(10 \mathrm{~mL})$ was added. Then the resulting mixture was stirred for $3 \mathrm{~min}$. The catalyst was separated by filtration, then washed with diethyl ether and dried at $120{ }^{\circ} \mathrm{C}$. The solvent was removed by distillation and the residue was washed with cold water. The pure product was obtained after recrystallization from $\mathrm{MeOH}$.

\subsection{Spectral data of the representative samples}

Compound 4b: IR (KBr) $v_{\max } / \mathrm{cm}^{-1}: 3285$ (s), 3200 (s), 1640 (s), 1605 (s); ${ }^{1} \mathrm{H}$ NMR (DMSO- $d_{6}$, $400 \mathrm{MHz}) \delta / \mathrm{ppm}: 0.91\left(3 \mathrm{H}, \mathrm{s}, \mathrm{CH}_{3}\right), 1.04\left(3 \mathrm{H}, \mathrm{s}, \mathrm{CH}_{3}\right), 1.97-2.01\left(1 \mathrm{H}, \mathrm{d}, J=16 \mathrm{~Hz}, \mathrm{CH}_{2}\right), 2.17-$ $2.19\left(1 \mathrm{H}, \mathrm{d}, J=8 \mathrm{~Hz}, \mathrm{CH}_{2}\right), 2.30-2.34\left(1 \mathrm{H}, \mathrm{d}, J=16 \mathrm{~Hz}, \mathrm{CH}_{2}\right), 2.47-2.51\left(1 \mathrm{H}, \mathrm{d}, J=16 \mathrm{~Hz}, \mathrm{CH}_{2}\right)$, $3.66\left(3 \mathrm{H}, \mathrm{s}, \mathrm{OCH}_{3}\right), 4.76(1 \mathrm{H}, \mathrm{s}, \mathrm{CH}), 7.07-6.86(\mathrm{~m}, 4 \mathrm{H}, \operatorname{Arom}), 7.76(1 \mathrm{H}, \mathrm{s}, \mathrm{NH}), 9.27(1 \mathrm{H}, \mathrm{s}$, $\mathrm{NH}) \mathrm{ppm} ;{ }^{13} \mathrm{C}$ NMR (DMSO-d, $\left.100 \mathrm{MHz}\right) \delta / \mathrm{ppm}: 27.34\left(\mathrm{CH}_{3}\right), 29.16\left(\mathrm{CH}_{3}\right), 31.56(\mathrm{C}), 32.75$ $\left(\mathrm{CH}_{2}\right), 50.52\left(\mathrm{CH}_{2}\right), 51.84(\mathrm{CH}), 55.29\left(\mathrm{OCH}_{3}\right), 107.84(\mathrm{C}), 113.39(\mathrm{CH}), 128.99(\mathrm{CH}), 135.15(\mathrm{C})$, 149.52 (C), 153.61 (C), 157.55 (CO), 194.86 (CO) ppm; Anal. Calcd. for $\mathrm{C}_{17} \mathrm{H}_{20} \mathrm{~N}_{2} \mathrm{O}_{3}$ : C, 67.98; $\mathrm{H}$, $6.71 ; \mathrm{N}, 9.33 \%$. Found: C, 68.228; H, 6.41; N, $9.25 \%$.

Compound 4c: IR (KBr) $v_{\max } / \mathrm{cm}^{-1}: 3443$ (s), 3192 (s), 1681 (s), 1665 (s), 1624 (s); ${ }^{1} \mathrm{H}$ NMR $\left(\mathrm{DMSO}-d_{6}, 400 \mathrm{MHz}\right) \delta / \mathrm{ppm}: 0.74\left(3 \mathrm{H}, \mathrm{s}, \mathrm{CH}_{3}\right), 0.81\left(3 \mathrm{H}, \mathrm{s}, \mathrm{CH}_{3}\right), 1.75-1.93\left(2 \mathrm{H}, \mathrm{m}, \mathrm{CH}_{2}\right), 2.09-$ $2.24\left(2 \mathrm{H}, \mathrm{m}, \mathrm{CH}_{2}\right), 5.34(1 \mathrm{H}, \mathrm{s}, \mathrm{CH}), 7.05-7.18(4 \mathrm{H}, \mathrm{m}, \operatorname{Arom}), 7.50(1 \mathrm{H}, \mathrm{s}, \mathrm{NH}), 9.33(1 \mathrm{H}, \mathrm{s}, \mathrm{NH})$ 
ppm; ${ }^{13} \mathrm{C}$ NMR (DMSO- $\left.d_{6}, 100 \mathrm{MHz}\right) \delta / \mathrm{ppm}: 28.64\left(\mathrm{CH}_{3}\right), 30.33(\mathrm{C}), 33.85\left(\mathrm{CH}_{2}\right), 51.38(\mathrm{CH})$, $107.41(\mathrm{C}), 128.99(\mathrm{CH}), 130.55(\mathrm{CH}), 131.01(\mathrm{CH}), 133.46(\mathrm{CH}), 142.79(\mathrm{CH}), 154.66(\mathrm{C}), 161.84$ (CO), 194.24 (CO) ppm; Anal. Calcd. for $\mathrm{C}_{16} \mathrm{H}_{17} \mathrm{ClN}_{2} \mathrm{O}_{2}$ : C, 63.05; H, 5.62; N, 9.19 \%. Found: C, $63.26 ; \mathrm{H}, 5.37$; N, $9.12 \%$.

Compound 4h: IR (KBr) $v_{\max } / \mathrm{cm}^{-1}: 3285$ (s), 3190 (s), 1646 (s), 1605 (s); ${ }^{1} \mathrm{H}$ NMR (DMSO- $d_{6}$, $400 \mathrm{MHz}) \delta / \mathrm{ppm}: 0.79\left(3 \mathrm{H}, \mathrm{s}, \mathrm{CH}_{3}\right), 0.879\left(3 \mathrm{H}, \mathrm{s}, \mathrm{CH}_{3}\right), 1.93-1.97\left(2 \mathrm{H}, \mathrm{d}, J=16 \mathrm{~Hz}, \mathrm{CH}_{2}\right), 2.00-$ $2.04\left(2 \mathrm{H}, \mathrm{d}, J=16 \mathrm{~Hz}, \mathrm{CH}_{2}\right), 2.26\left(3 \mathrm{H}, \mathrm{s}, \mathrm{CH}_{3}\right), 4.50(1 \mathrm{H}, \mathrm{s}, \mathrm{CH}), 6.80-6.98(4 \mathrm{H}, \mathrm{m}$, Arom $), 7.06$ $(2 \mathrm{H}$, br, $\mathrm{NH})$ ppm; Anal. Calcd. for $\mathrm{C}_{17} \mathrm{H}_{20} \mathrm{~N}_{2} \mathrm{O}_{2}: \mathrm{C}, 71.81 ; \mathrm{H}, 7.09 ; \mathrm{N}, 9.85 \%$. Found: C, 72.03; H, $6.90 ; \mathrm{N}, 9.72 \%$.

Compound 4j: IR (KBr) $v_{\max } / \mathrm{cm}^{-1}: 3228$ (s), 2962 (s), 1649 (s), 1620 (s); ${ }^{1} \mathrm{H}$ NMR (DMSO- $d_{6}$, $400 \mathrm{MHz}) \delta / \mathrm{ppm}: 1.890-1.833\left(2 \mathrm{H}, \mathrm{m}, \mathrm{CH}_{2}\right), 1.94-2.00\left(2 \mathrm{H}, \mathrm{m}, \mathrm{CH}_{2}\right), 2.306-2.347\left(2 \mathrm{H}, \mathrm{m}, \mathrm{CH}_{2}\right)$, $4.594(1 \mathrm{H}, \mathrm{s}, \mathrm{CH}), 7.096-7.241\left(5 \mathrm{H}, \mathrm{m}\right.$, Arom),7.516 (1H, s, NH), $9.347(1 \mathrm{H}, \mathrm{s}, \mathrm{NH}) \mathrm{ppm} ;{ }^{13} \mathrm{C} \mathrm{NMR}$ (DMSO-d $\left.d_{6}, 100 \mathrm{MHz}\right) \delta / \mathrm{ppm}: 21.00\left(\mathrm{CH}_{2}\right), 27.77\left(\mathrm{CH}_{2}\right), 36.75\left(\mathrm{CH}_{2}\right), 50.94(\mathrm{CH}), 107.69(\mathrm{C})$, $113.39(\mathrm{CH}), 129.03(\mathrm{CH}), 136.07(\mathrm{C}), 149.51(\mathrm{C}), 154.81$ (C), $158.74(\mathrm{CO}), 191.82$ (CO) ppm; Anal. Calcd. for $\mathrm{C}_{14} \mathrm{H}_{14} \mathrm{~N}_{2} \mathrm{O}_{2}$ : C, 69.41; H, 5.82; N, $11.56 \%$. Found: C, 69.22; H, 5.92; N, $11.41 \%$.

Compound 4n: IR (KBr) $v_{\max } / \mathrm{cm}^{-1}: 3302$ (s), 3190 (s), 1688 (s), 1667 (s), 1636 (s); ${ }^{1} \mathrm{H}$ NMR $\left(\mathrm{DMSO}-d_{6}, 400 \mathrm{MHz}\right) \delta / \mathrm{ppm}: 1.35-1.61\left(2 \mathrm{H}, \mathrm{m}, \mathrm{CH}_{2}\right), 1.74-1.91\left(2 \mathrm{H}, \mathrm{m}, \mathrm{CH}_{2}\right), 1.96-2.18(2 \mathrm{H}, \mathrm{m}$, $\left.\mathrm{CH}_{2}\right), 3.50\left(3 \mathrm{H}, \mathrm{s}, \mathrm{OCH}_{3}\right), 4.39(1 \mathrm{H}, \mathrm{s}, \mathrm{CH}), 6.56-6.65(4 \mathrm{H}, \mathrm{m}, \mathrm{Arom}), 6.96(1 \mathrm{H}, \mathrm{s}, \mathrm{NH}), 8.11(1 \mathrm{H}, \mathrm{s}$, $\mathrm{NH}) \mathrm{ppm} ;{ }^{13} \mathrm{C}$ NMR (DMSO-d $\left.d_{6} 100 \mathrm{MHz}\right) \delta / \mathrm{ppm}: 20.99\left(\mathrm{CH}_{2}\right), 29.00\left(\mathrm{CH}_{2}\right), 37.27(\mathrm{CH} 2)$, 55.02(CH), $101.62(\mathrm{C}), 110.42(\mathrm{CH}), 111.48(\mathrm{CH}), 119.84(\mathrm{CH}), 126.56(\mathrm{CH}), 129.25(\mathrm{CH}), 131.70$ $(\mathrm{CH}), 156.55(\mathrm{C}), 169.11(\mathrm{CO}), 196.02(\mathrm{CO}) \mathrm{ppm}$; Anal. Calcd. for $\mathrm{C}_{15} \mathrm{H}_{16} \mathrm{~N}_{2} \mathrm{O}_{3}$ : C, 66.16; H, 5.92; N, $10.29 \%$. Found: C, 66.40; H, 5.752; N, $10.11 \%$.

Compound 4q: IR(KBr) $v_{\max } / \mathrm{cm}^{-1}: 3437$ (s), 3305 (s), 1664 (s), 1613 (s), 1589 (s); ${ }^{1} \mathrm{H}$ NMR $\left(\mathrm{DMSO}-d_{6}, 400 \mathrm{MHz}\right) \delta / \mathrm{ppm}: 0.679\left(3 \mathrm{H}, \mathrm{s}, \mathrm{CH}_{3}\right), 0.803\left(3 \mathrm{H}, \mathrm{s}, \mathrm{CH}_{3}\right), 1.82-2.01\left(2 \mathrm{H}, \mathrm{m}, \mathrm{CH}_{2}\right)$, 2.06-2.22 (2H, m, $\left.\mathrm{CH}_{2}\right), 4.94(1 \mathrm{H}, \mathrm{s}, \mathrm{CH}), 7.06-7.16(4 \mathrm{H}, \mathrm{m}$, Arom $), 7.61(1 \mathrm{H}, \mathrm{s}, \mathrm{NH}), 9.35(1 \mathrm{H}, \mathrm{s}$, NH) ppm; ${ }^{13} \mathrm{C}$ NMR (DMSO-d, $\left.100 \mathrm{MHz}\right) \delta / \mathrm{ppm}: 27.28\left(\mathrm{CH}_{3}\right), 29.12(\mathrm{C}), 32.78\left(\mathrm{CH}_{2}\right), 50.21$ $\left(\mathrm{CH}_{2}\right), 52.08(\mathrm{CH}), 107.25(\mathrm{C}), 125.32(\mathrm{CH}), 126.25(\mathrm{CH}), 127.35(\mathrm{CH}), 130.48(\mathrm{CH}), 133.26(\mathrm{CH})$, $145.90(\mathrm{CH}), 147.42(\mathrm{C}), 158.11(\mathrm{CO}), 193.03(\mathrm{CO}) \mathrm{ppm}$; Anal. Calcd. for $\mathrm{C}_{16} \mathrm{H}_{17} \mathrm{ClN}_{2} \mathrm{O}_{2}$ : C, 63.05; H, 5.62; N, $9.19 \%$. Found: C, 63.22; H, 5.55; N, $9.08 \%$.

Compound 4r: IR (KBr) $v_{\max } / \mathrm{cm}^{-1}: 3278$ (s), 3162 (s), 1642 (s), 1572 (s); ${ }^{1} \mathrm{H}$ NMR (DMSO-d 6 , $400 \mathrm{MHz}) \delta / \mathrm{ppm}: 0.903\left(3 \mathrm{H}, \mathrm{s}, \mathrm{CH}_{3}\right), 1.041\left(3 \mathrm{H}, \mathrm{s}, \mathrm{CH}_{3}\right), 2.053-2.093\left(2 \mathrm{H}, \mathrm{m}, \mathrm{CH}_{2}\right), 2.212(2 \mathrm{H}$, s, $\left.\mathrm{CH}_{2}\right), 2.247\left(3 \mathrm{H}, \mathrm{s}, \mathrm{CH}_{3}\right), 5.137(1 \mathrm{H}, \mathrm{s}, \mathrm{CH}), 7.091-7.154(\mathrm{~m}, 4 \mathrm{H}$, Arom $), 9.657(1 \mathrm{H}, \mathrm{s}, \mathrm{NH})$, 10.559(1H, s, NH) ppm; ${ }^{13} \mathrm{C}$ NMR (DMSO-d $\left.6,100 \mathrm{MHz}\right) \delta / \mathrm{ppm}: 20.33\left(\mathrm{CH}_{3}\right), 27.34\left(\mathrm{CH}_{3}\right), 29.60$ $\left(\mathrm{CH}_{3}\right), 32.75(\mathrm{C}), 37.84(\mathrm{CH} 2), 50.75(\mathrm{CH} 2), 51.51(\mathrm{CH}), 105.84(\mathrm{C}), 123.29(\mathrm{CH}), 129.00(\mathrm{CH})$, 137.37 (C), 140.00 (C), 149.47 (C), 174.61 (CS), 195.06 (CO) ppm; Anal. Calcd. for $\mathrm{C}_{17} \mathrm{H}_{20} \mathrm{~N}_{2} \mathrm{OS}$ : C, 67.97; H, 6.71; N, 9.32; S, 10.67 \%. Found: C, 68.18; H, 6.50; N, 9.21; S, $10.45 \%$.

Compound 4t: IR (KBr) $v_{\max } / \mathrm{cm}^{-1}: 3262$ (s), 3165 (s), 1666 (s), 1641 (s), 1584 (s); ${ }^{1} \mathrm{H}$ NMR $\left(\mathrm{DMSO}-d_{6}, 400 \mathrm{MHz}\right) \delta / \mathrm{ppm}: 0.909\left(3 \mathrm{H}, \mathrm{s}, \mathrm{CH}_{3}\right), 1.033\left(3 \mathrm{H}, \mathrm{s}, \mathrm{CH}_{3}\right), 2.086-2.219\left(2 \mathrm{H}, \mathrm{m}, \mathrm{CH}_{2}\right)$, 2.399-2.431 (2H, m, $\left.\mathrm{CH}_{2}\right), 3.729\left(3 \mathrm{H}, \mathrm{s}, \mathrm{OCH}_{3}\right), 5.122(1 \mathrm{H}, \mathrm{s}, \mathrm{CH}), 6.888-6.910(2 \mathrm{H}, \mathrm{m}$, Arom $)$, 7.146-7.124 (2H, m, Arom), $9.640(1 \mathrm{H}, \mathrm{s}, \mathrm{NH}), 10.546(1 \mathrm{H}, \mathrm{s}, \mathrm{NH}) \mathrm{ppm} ;{ }^{13} \mathrm{C}$ NMR (DMSO- $d_{6}, 100$ MHz) $\delta / \mathrm{ppm}: 27.25(\mathrm{CH} 3), 29.29\left(\mathrm{CH}_{3}\right), 32.73(\mathrm{C}), 38.93\left(\mathrm{CH}_{2}\right), 50.31\left(\mathrm{CH}_{2}\right), 52.09(\mathrm{CH}), 55.56$ $\left(\mathrm{OCH}_{3}\right), 108.75(\mathrm{C}), 114.27(\mathrm{CH}), 128.09(\mathrm{CH}), 136.06(\mathrm{C}), 148.92(\mathrm{C}), 159.09(\mathrm{C}), 174.82(\mathrm{CS})$, 194.11 (CO) ppm; Anal. Calcd. for $\mathrm{C}_{17} \mathrm{H}_{20} \mathrm{~N}_{2} \mathrm{O}_{2} \mathrm{~S}: \mathrm{C}, 64.53 ; \mathrm{H}, 6.37 ; \mathrm{N}, 8.85 ; \mathrm{S}, 10.13$ \%. Found: C, $64.61 ; \mathrm{H}, 6.39 ; \mathrm{N}, 8.72 ; \mathrm{S}, 9.90 \%$.

Compound 4u: IR (KBr) $v_{\max } / \mathrm{cm}^{-1}: 3262$ (s), 3173 (s), 1698 (s), 1620 (s), 1567 (s); ${ }^{1} \mathrm{H}$ NMR 
(DMSO- $\left.d_{6}, 400 \mathrm{MHz}\right) \delta / \mathrm{ppm}: 0.897\left(3 \mathrm{H}, \mathrm{s}, \mathrm{CH}_{3}\right), 1.035\left(3 \mathrm{H}, \mathrm{s}, \mathrm{CH}_{3}\right), 2.059-2.229\left(2 \mathrm{H}, \mathrm{m}, \mathrm{CH}_{2}\right)$, 2.364-2.484 (2H, m, $\left.\mathrm{CH}_{2}\right), 5.193(1 \mathrm{H}, \mathrm{s}, \mathrm{CH}), 7.223-7.280(\mathrm{~m}, 3 \mathrm{H}$, Arom $), 7.328-7.365(2 \mathrm{H}, \mathrm{m}$, Arom), $9.696(1 \mathrm{H}, \mathrm{s}, \mathrm{NH}), 10.594(1 \mathrm{H}, \mathrm{s}, \mathrm{NH}) \mathrm{ppm} ;{ }^{13} \mathrm{C}$ NMR (DMSO-d $\left.6,100 \mathrm{MHz}\right) \delta / \mathrm{ppm}: 27.24$ $\left(\mathrm{CH}_{3}\right), 29.28(\mathrm{C}), 32.73\left(\mathrm{CH}_{2}\right), 50.31\left(\mathrm{CH}_{2}\right), 52.69(\mathrm{CH}), 108.61(\mathrm{C}), 126.89(\mathrm{CH}), 128.02(\mathrm{CH})$, $128.96(\mathrm{CH}), 143.83(\mathrm{CH}), 149.16(\mathrm{C}), 175.08(\mathrm{CS}), 194.12(\mathrm{CO}) \mathrm{ppm} ;$ Anal. Calcd. for $\mathrm{C}_{16} \mathrm{H}_{18} \mathrm{~N}_{2} \mathrm{OS}$ : C, 67.10; H, 6.33; N, 9.78; S, 11.20 \%. Found: C, 67.22; H, 6.40; N, 9.65; S, $11.35 \%$.

\section{Acknowledgements}

The authors gratefully acknowledge partial support of this work by Yasouj University, Iran.

\section{References}

1. Alavi Nikje M. M., Khanmohammadi M. R., Bagheri A., and Haghshenas M. (2012) Nanosilica reinforced epoxy floor coating composites: preparation and thermophysical characterization Curr. Chem. Lett., 1, 13-20.

2. Khodabakhshi S., and Karami B. (2012) A rapid and eco-friendly synthesis of novel and known benzopyrazines using silica tungstic acid (STA) as a new and recyclable catalyst. Catal. Sci. Technol., 2, 1940-1944.

3. Corma A., and Garcia H. (2006) Silica-bound homogenous catalysts as recoverable and reusable catalysts in organic synthesis. Adv. Synth. Catal., 348, 1391-1412.

4. Rodriguez, N. M., Kim M-S., and Baker R. T. K. (1994) Carbon nanofibers: a unique catalyst support medium. J. Phys. Chem., 98, 13108-13111.

5. Balazsik K., Szori K., Szollosi G., and Bartok M. (2011) New phenomenon in competitive hydrogenation of binary mixtures of activated ketones over unmodified and cinchonidinemodified Pt/alumina catalyst. Catal. Commun. 12, 1410-1414.

6. Kantam M. L., Jaya, V. S., Lakshmi M. J., Reddy B. R., Choudary B. M., and Bhargava S. K. (2007) Alumina supported copper nanoparticles for aziridination and cyclopropanation reactions. Catal. Commun., 8, 1963-1968.

7. Szollosi G., Makra Z., Fulop F., and Bartok M. (2011) The first case of competitive heterogeneously catalyzed hydrogenation using continuous-flow fixed-bed reactor system: hydrogenation of binary mixtures of activated ketones on Pt-alumina and on Pt-aluminacinchonidine catalysts. Catal. Lett., 141, 1616-1620.

8. Gila W., Boczon K., Trzeciak A. M., Ziołkowski J. J., Garcia-Verdugo E., Luis S. V., and Sans V. (2009) Supported N-heterocyclic carbene rhodium complexes as highly selective hydroformylation catalysts. J. Mol. Catal. A: Chem., 309, 131-136.

9. Miao J., Wan H., Shao Y., Guan G., and Xu B. (2011) Acetalization of carbonyl compounds catalyzed by acidic ionic liquid immobilized on silica gel. J. Mol. Catal. A: Chem., 348, 77-82.

10. Held A., Kowalska-Kus J., and Nowinska K. (2012) Epoxidation of propene on vanadium species supported on silica supports of different structure. Catal. Commun., 17, 108-113.

11. Zhao S., Zhao J., Lou L.-L., Liu S. (2011) Silica-supported quinine-CuBr catalyzed atom transfer radical polymerization of methyl methacrylate. Catal. Commun., 12, 1027-1030.

12. Chen K., Wang K., Kirichian A. M., Al Aowad A. F., Iyer L. K., Adelstein S. J., Kassism A. I. (2006) In silico design, synthesis, and biological evaluation of radioiodinated quinazolinone derivatives for alkaline phosphatase-mediated cancer diagnosis and therapy. Mol. Cancer Ther., 5, 3001-3013.

13. Tonkikh N. N., Strakovs A., and Petrova M. V. (2004) Multicomponent synthesis of 2,5-dioxoand 4-aryl-5-oxo-2-thioxo-1,2,3,4,5,6,7,8-octahydroquinazolines. Chem. Heterocycl. Compd., 40, 43-46. 
14. Hassani Z., Islami M. R., and Kalantari M. (2006) An efficient one-pot synthesis of octahydroquinazolinone derivatives using catalytic amount of $\mathrm{H}_{2} \mathrm{SO}_{4}$ in water. Bio. Org. Med. Chem. Lett., 16, 4479-4482.

15. Kantevari S., Bantu R., and Nagarapu L. (2006) TMSCl mediated highly efficient one-pot synthesis of octahydroquinazolinone and 1,8-dioxo-octahydroxanthene derivatives. Arkivoc (xvi), 136-148.

16. Sabitha G., Reddy G. S. K., Reddy K. B., and Yadav J. S. (2003) Vanadium(III) chloride catalyzed Biginelli condensation: solution phase library generation of dihydropyrimidin- $(2 \mathrm{H})-$ ones. Tetrahedran Lett., 44, 6497-6499.

17. Candan M. M., Kendi E., Yarim M., Sarac S., and Ertan M., (2001) Crystal structure of 4-(4bromophenyl)-1,7,7-trimethyl-1,2,3,4,5,6,7,8-octahydroquinazoline-2,5-dione. Anal. Sci., 17, 1023-1024.

18. Kidwai M., Saxena S., Khan M. K. R., Thukral S. S. (2005) Synthesis of 4-aryl-7,7-dimethyl$1,2,3,4,5,6,7,8$-octahydroquinazoline-2-one/thione-5-one derivatives and evaluation as antibacterials. Eur. J. Med. Chem., 40, 816-819.

19. Karami B., Ghashghaee V. and Khodabakhshi S. (2012) Novel silica tungstic acid (STA): Preparation, characterization and its first catalytic application in synthesis of new benzimidazoles. Catal. Commun., 20, 71-75.

20. Karami B., and Kiani M. (2011) $\mathrm{ZrOCl}_{2} \cdot 8 \mathrm{H}_{2} \mathrm{O} / \mathrm{SiO}_{2}$ : An efficient and recyclable catalyst for the preparation of coumarin derivatives by Pechmann condensation reaction. Catal. Commun., 14, 62-67.

21. Karami B., Khodabakhshi S., and Nikrooz M. (2011) Synthesis of aza-polycyclic compounds: novel phenazines and quinoxalines using molybdate sulfuric acid (MSA). Polycyclic Aromat. Compds., 31, 97-109.

22. Karami B., Khodabakhshi, S., Safikhani, N., Arami A. (2012) A green and highly efficient solvent-free synthesis of novel calicx[4]resorcinarene derivatives using tungstate sulfuric acid. Bull. Korean Chem. Soc., 33, 123-127.

23. Karami B., Haghighijoua Z., Farahi M., and Khodabakhshi S. (2012) One-Pot synthesis of dihydropyrimidine-thione derivatives using tungstate sulfuric acid (TSA) as a recyclable catalyst. Phosphorus Sulfur Silicon Relat. Elem., 187, 754-761.

24. Karami B., Rooydel R., and Khodabakhshi, S. (2011) A rapid synthesis of some 1,4-aryldiazines by the use of lithium chloride as an effective catalyst. Acta Chim Slov., 59, 183-188.

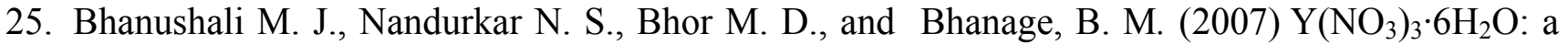
novel and reusable catalyst for one pot synthesis of 3,4-dihydropyrimidin-2(1H)-ones under solvent-free conditions. J. Mol. Catal. A: Chem., 271, 14-17.

26. Zhang H., Guo X., Zhang Q., Ma Y., Zhou H., Li J., Wang L., and Deng Y. (2008) Synthesis of dialkyl hexamethylenedicarbamate from 1,6-hexamethylenediamine and alkyl carbamate over $\mathrm{Y}\left(\mathrm{NO}_{3}\right)_{3} \cdot 6 \mathrm{H}_{2} \mathrm{O}$ catalyst. J. Mol. Catal. A: Chem., 296, 36-41.

27. Kantam M. L., Rao B. P. C., Reddy R. S., Sekhar N. S., Sreedhar B., and Choudary B. M. (2007) Aerobic epoxidation of olefins catalyzed by $\mathrm{Co}-\mathrm{SiO}_{2}$ nanocomposites. J. Mol. Catal. A: Chem., 272, $1-5$.

28. Narayanan S., and Prasad B. P. (1995) Characterization and aniline alkylation activity of vanadia and silica-supported vanadia catalysts. J. Mol. Catal. A: Chem., 96, 57-64.

29. Borah R., Dutta P., and Sarma P. (2013) Investigation of efficient synthesis of 1, 8-dioxooctahydroxanthene derivatives under solvent-free grinding method. Curr. Chem. Lett., 2, 159166. 